\section{Learning to live with lasers}

\section{Lasers: Harnessing the Atom's \\ Light}

by James P. Harbison and Robert E. Nahory W. H. Freeman: 1998. Pp. 214. \$34.95, £22.95

\section{J.Christopher Whitehead}

The laser was born in 1960 and one might think it should now be approaching middle age gracefully. But the pace at which new laser systems are being developed and new applications for lasers discovered suggests that is is undergoing a continual rebirth into a state of sophisticated maturity. Lasers are part of everybody's life yet we are often unaware of their existence, despite encountering them at the supermarket checkout and in the compact disc player, and relying on their power to bring high-speed communication into our homes.

James Harbison and Robert Nahory have produced an elegant book describing the many aspects of the laser, from its history and basic science to recent developments. Much of the book deals with the semiconductor lasers important in the field of high-speed communications. These lasers can be built to order by producing different layers of material only an atom or two thick. The true multidisciplinary nature of laser development is revealed as the authors describe the techniques of growing the crystals, the epitaxial methods of depositing the layers, and the processes of etching to remove selected parts of the device; this task involves scientists and engineers with skills in physics, chemistry, materials science and electrical engineering.

The trend is towards the microlaser, a million of which can be grown on a chip the size of a small fingernail. Quantum dot lasers have an active region consisting of a dot of indium gallium arsenide that is only 28 nanometres in diameter and 3 nanometres high. These devices are extremely compact, cheap to produce and use very litthe electrical power. At this scale, one can imagine such lasers being used in areas beyond communications, including optical computers and three-dimensional displays.

Harbison and Nahory also describe developments in lasers much larger in scale. These include X-ray lasers, which can be used to produce holograms of living cells by freezing an image in a billionth of a second; vacuum ultraviolet lasers, which can clean old paintings or 're-machine' the lens in a human eye to correct vision problems; lasers that emit short bursts of light lasting only a few femtoseconds, which can be used to investigate photosynthesis at a molecular level; and ultra-high-power lasers, in which a pulse from a bank oflasers is focused into a tiny pellet containing deuterium and tritium, creating pressures and temperatures akin to those

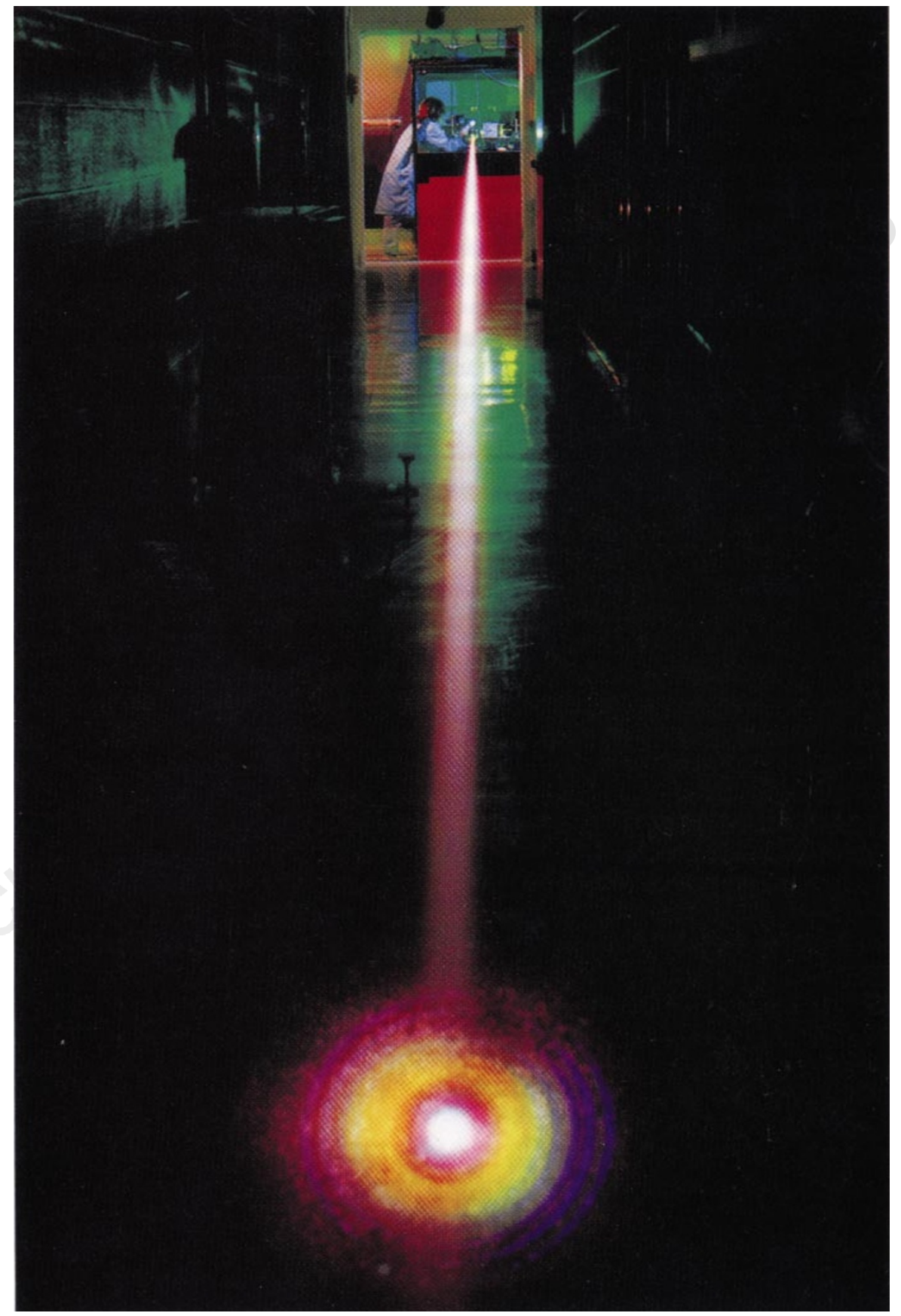

Tomorrow's world: a 40-metre plasma channel created by an ultrashort laser pulse, University of Michigan. Ultrashort pulse lengths could make tabletop X-ray lasers a reality.

at the centre of a massive star releasing energy in the process of laser fusion. They discuss the exciting ways in which lasers will aid understanding of many fundamental processes and provide solutions and opportunities that will transform the lives of many.

The authors understand the difficulties that non-specialists may have in understanding the concepts they discuss, and are imaginative in devising helpful analogies. They do this with the aid of first-class illustrations that make the book accessible to anyone with a background in science.

J. Christopher Whitehead is in the Chemistry

Department, University of Manchester, Manchester M13 9PL, UK.

\section{New in paperback}

\section{In Search of the Big Bang: The Life and Death of the Universe}

By John Gribbin

Penguin $£ 8.99$

Updated edition

By Bettyan Holtzman Kevles

Helix/Addison-Wesley, $\$ 18$

Reviewed by J. Mallard in Nature 386, 567 (1997).

\section{Naked to the Bone: Medical Imaging in the Twentieth Century}

